\title{
Ascent and descent for sets of operators
}

\author{
by \\ DereK Kitson (Dublin)
}

\begin{abstract}
We extend the notion of ascent and descent for an operator acting on a vector space to sets of operators. If the ascent and descent of a set are both finite then they must be equal and give rise to a canonical decomposition of the space. Algebras of operators, unions of sets and closures of sets are treated. As an application we construct a Browder joint spectrum for commuting tuples of bounded operators which is compactvalued and has the projection property.
\end{abstract}

Introduction. In this paper we formulate the definitions and basic properties of ascent and descent for an arbitrary (non-empty) set $A$ of operators acting on a vector space $X$ over any field $\mathbb{K}$. This notion of ascent and descent extends the classical theory for single operators (see for example $[3,6,8,9])$ and the work of Grunenfelder and Omladič ([2]) which deals with commuting tuples. Much of the initial theory is purely algebraic but Sections 2, 3 and 4 are also concerned with bounded operators acting on a real or complex Banach space. By operator we will always mean a linear map and denote by $L(X)$ the set of all operators acting on $X$. The set of bounded operators acting on a Banach space $X$ is denoted $B(X)$.

In Section 1, we begin by introducing ascending and descending chain lengths for a set of operators. Unlike the case of a single operator, it is possible for a set to have finite ascending and descending chain lengths which are not equal. We then define the stronger notions of ascent and descent for a set of operators. If the ascent and descent of a set are both finite then they must be equal and in this case must also equal the ascending and descending chain lengths. We show that having finite ascent and descent is equivalent to the existence of a decomposition $X=X_{1} \oplus X_{2}$ where $X_{1}$ and $X_{2}$ are invariant under each operator in the set and such that the restriction of the set to $X_{1}$ is nilpotent while the restriction to $X_{2}$ is bijective (in an appropriate sense). The spaces $X_{1}, X_{2}$ are uniquely determined by the ascent and descent of $A$.

2000 Mathematics Subject Classification: Primary 47A13, 47A46; Secondary 47A05. Key words and phrases: Browder tuple, Browder joint spectrum. 
In Section 2, we consider the behaviour of ascent and descent under the operations of taking unions, generating algebras and (in the case of bounded operators on a Banach space) forming closures of sets. Section 3 deals with tuples of operators. In this case, ascent and descent may be expressed in terms of associated row and column operators. We show that complete duality holds for tuples of bounded operators which satisfy a Fredholm condition. Finally, in Section 4, we construct a joint spectrum for commuting tuples of bounded operators which extends the Browder essential spectrum. We show that this is a compact-valued joint spectrum which has the projection property and we provide a characterisation analogous to that of D. C. Lay ([5]). This Browder joint spectrum is smaller than the Taylor-Browder spectrum considered by Curto and Dash ([1]) but contains the upper and lower semiBrowder spectra of Kordula, Müller and Rakočević ([4]).

A subspace $Y$ of $X$ which is invariant under each $a \in A$ will be called $A$-invariant. Given an $A$-invariant subspace $Y$, we denote by $\left.A\right|_{Y}$ the set $\left\{\left.a\right|_{Y}: a \in A\right\}$ of operators on $Y$. We denote by Ker $a$ and Ran $a$ the kernel and range respectively of an operator $a$.

1. Ascent and descent. Let $A \subseteq L(X)$ be a non-empty set of operators acting on a vector space $X$. We define the null space and range space of $A$ respectively as

$$
N(A)=\bigcap_{a \in A} \operatorname{Ker} a, \quad R(A)=\operatorname{span}\left(\bigcup_{a \in A} \operatorname{Ran} a\right) .
$$

For each $k \in \mathbb{N}$ we define $A^{k}=\left\{a_{1} \ldots a_{k}: a_{1}, \ldots, a_{k} \in A\right\}$ and $A^{0}=\{I\}$ where $I$ denotes the identity operator on $X$.

Lemma 1.1. $N\left(A^{k}\right)$ and $R\left(A^{k}\right)$ are $A$-invariant subspaces of $X$ for each $k \in \mathbb{N}$.

Proof. This is straightforward for $N\left(A^{k}\right)$. Now, $\bigcup_{a_{1}, \ldots, a_{k} \in A}\left(\operatorname{Ran} a_{1} \ldots a_{k}\right)$ is clearly invariant under each $a \in A$. By definition $R\left(A^{k}\right)$ is the linear span of this set and so $R\left(A^{k}\right)$ is an $A$-invariant subspace of $X$.

It is not too difficult to see that we have an increasing chain of null spaces,

$$
\{0\}=N\left(A^{0}\right) \subseteq N(A) \subseteq N\left(A^{2}\right) \subseteq \cdots,
$$

and a decreasing chain of range spaces,

$$
X=R\left(A^{0}\right) \supseteq R(A) \supseteq R\left(A^{2}\right) \supseteq \cdots .
$$

Definition 1.2. The ascending chain length of $A$, denoted $\operatorname{acl}(A)$, is the smallest non-negative integer $r$ such that $N\left(A^{r}\right)=N\left(A^{r+1}\right)$. The descending chain length of $A$, denoted $\operatorname{dcl}(A)$, is the smallest non-negative integer $r$ 
such that $R\left(A^{r}\right)=R\left(A^{r+1}\right)$. In the case where no such $r$ exists we say the ascending or descending chain length of $A$ is infinite.

Proposition 1.3.

(i) If $N\left(A^{r}\right)=N\left(A^{r+1}\right)$ for some $r \geq 0$ then $N\left(A^{r}\right)=N\left(A^{s}\right)$ for all $s \geq r$.

(ii) If $R\left(A^{r}\right)=R\left(A^{r+1}\right)$ for some $r \geq 0$ then $R\left(A^{r}\right)=R\left(A^{s}\right)$ for all $s \geq r$.

Proof. Apply a straightforward induction argument.

REMARK 1.4. In the case of a single operator, if the ascending and descending chain lengths are both finite then they are equal ([9, Theorem V.6.2]). The following multishift example from [4] shows that this is not true in general for sets containing two or more operators, even when the operators commute.

EXAMPLE 1.5. Let $H$ be the complex Hilbert space with orthonormal basis $\left\{e_{i, j}:(i, j) \in S\right\}$ where $S=\{(i, j) \in \mathbb{Z} \times \mathbb{Z}: i \geq 0$ or $j \geq 0\} \cup$ $\{(-1,-1)\}$. Let $A=\left\{a_{1}, a_{2}\right\}$ where $a_{1}$ and $a_{2}$ are commuting operators on $H$ given by $a_{1}\left(e_{i, j}\right)=e_{i+1, j}$ and $a_{2}\left(e_{i, j}\right)=e_{i, j+1}$. Both $a_{1}$ and $a_{2}$ are injective and so the ascending chain length of $A$ is 0 . However, the descending chain length of $A$ is 1 . To see this, note that for $k \geq 1, R\left(A^{k}\right)$ is the closed subspace of $H$ spanned by $\left\{e_{i, j}: i \geq 0\right.$ or $\left.j \geq 0\right\}$.

Definition 1.6. Let $A$ be a non-empty set of operators on $X$. The ascent of $A$, denoted $\alpha(A)$, is the smallest non-negative integer $r$ such that $N(A) \cap R\left(A^{r}\right)=\{0\}$. The descent of $A$, denoted $\delta(A)$, is the smallest nonnegative integer $r$ such that $N\left(A^{r}\right)+R(A)=X$. In the case that no such $r$ exists we say that $A$ has infinite ascent or infinite descent.

Proposition 1.7. If $a \in L(X)$ then $\alpha(a)=\operatorname{acl}(a)$ and $\delta(a)=\operatorname{dcl}(a)$.

Proof. Apply [9, Theorems V.6.3 and V.6.4].

Proposition 1.8. Let $r$ be a non-negative integer.

(i) If $N(A) \cap R\left(A^{r}\right)=\{0\}$ then $N\left(A^{k}\right) \cap R\left(A^{r}\right)=\{0\}$ for all $k \geq 1$.

(ii) If $N\left(A^{r}\right)+R(A)=X$ then $N\left(A^{r}\right)+R\left(A^{k}\right)=X$ for all $k \geq 1$.

Proof. The results follow by an induction argument.

Proposition 1.9.

(i) $\operatorname{acl}(A) \leq \alpha(A)$ and $\operatorname{dcl}(A) \leq \delta(A)$.

(ii) If $\alpha(A)<\infty$ then $\alpha(A) \leq \operatorname{dcl}(A)$.

(iii) If $\delta(A)<\infty$ then $\delta(A) \leq \operatorname{acl}(A)$.

(iv) If $\alpha(A), \delta(A)<\infty$ then $\alpha(A)=\operatorname{acl}(A)=\operatorname{dcl}(A)=\delta(A)$. 
Proof. (i) Suppose $A$ has finite ascent $r$ and let $x \in N\left(A^{r+1}\right)$. Then for all $a \in A$ we have $a\left(a_{1} \ldots a_{r} x\right)=0$ and so $a_{1} \ldots a_{r} x \in N(A) \cap R\left(A^{r}\right)$ for all $a_{1}, \ldots, a_{r} \in A$. Now $N(A) \cap R\left(A^{r}\right)=\{0\}$ and so $x \in N\left(A^{r}\right)$. Since $N\left(A^{r}\right)$ is contained in $N\left(A^{r+1}\right)$ we have shown that $N\left(A^{r}\right)=N\left(A^{r+1}\right)$. Thus the ascending chain length of $A$ is at most $r$. The second inequality holds by a similar argument.

(ii) Suppose $\alpha(A)<\infty$. If $A$ has descending chain length $s<\alpha(A)$ then by Proposition 1.3, $R\left(A^{s}\right)=R\left(A^{\alpha(A)}\right)$ and so $N(A) \cap R\left(A^{s}\right)=\{0\}$. But this contradicts the minimality of the ascent and so the result follows. A similar argument holds for (iii).

(iv) follows immediately on combining (i)-(iii).

REMARK 1.10. Note that by Proposition 1.9, finite ascent and descent implies finite chain lengths. The converse is not true in general. In Example 1.5, $A$ has finite chain lengths but the descent of $A$ is infinite.

Definition 1.11. We will say a set $A \subseteq L(X)$ is nilpotent if there exists $k \in \mathbb{N}$ with $A^{k}=\{0\}$. We will call $A$ injective if $N(A)=\{0\}$, surjective if $R(A)=X$ and bijective if it is both injective and surjective.

THEOREM 1.12. A has finite ascent and finite descent if and only if there exist $A$-invariant subspaces $X_{1}, X_{2}$ of $X$ such that $X=X_{1} \oplus X_{2},\left.A\right|_{X_{1}}$ is nilpotent and $\left.A\right|_{X_{2}}$ is bijective. Moreover, the spaces $X_{1}$ and $X_{2}$ are given uniquely by $X_{1}=N\left(A^{r}\right)$ and $X_{2}=R\left(A^{r}\right)$, where $r$ is the common value of the ascent and descent of $A$.

Proof. Suppose $A$ has finite ascent and finite descent. By Proposition 1.9, the ascent and descent of $A$ are equal. If $r$ is their common value then let $X_{1}=N\left(A^{r}\right)$ and $X_{2}=R\left(A^{r}\right)$. Note that $X_{1}$ and $X_{2}$ are $A$-invariant subspaces of $X$ and by Proposition 1.8, $X=X_{1} \oplus X_{2}$. Now, $\left.A\right|_{X_{1}}$ is nilpotent since $\left.A^{r}\right|_{X_{1}}=\{0\}$. Also, $N(A) \cap R\left(A^{r}\right)=\{0\}$ and so $\left.A\right|_{X_{2}}$ is injective. That $\left.A\right|_{X_{2}}$ is surjective follows since $N\left(A^{r}\right)+R(A)=X$.

For the converse, suppose there exist $A$-invariant subspaces $X_{1}, X_{2}$ of $X$ as in the statement of the theorem. Since $\left.A\right|_{X_{1}}$ is nilpotent there exists $k \in \mathbb{N}$ such that $X_{1} \subseteq N\left(A^{k}\right)$ and $R\left(A^{k}\right) \subseteq X_{2}$. Now $\left.A\right|_{X_{2}}$ is injective and so $N(A) \cap R\left(A^{k}\right)=\{0\}$. Also, $\left.A\right|_{X_{2}}$ is surjective and so $N\left(A^{k}\right)+R(A)=X$. Hence $A$ has ascent and descent at most $k$. By the above argument, $X=$ $N\left(A^{r}\right) \oplus R\left(A^{r}\right)$ where $r=\alpha(A)=\delta(A)$. Now, $A$ has ascending chain length $r$ and so $X_{1} \subseteq N\left(A^{r}\right)$. Since $\left.A\right|_{X_{2}}$ is surjective we have (by Proposition 1.3), $X_{2}=R\left(\left.A^{r}\right|_{X_{2}}\right)=R\left(A^{r}\right)$. We conclude that $X_{1}=N\left(A^{r}\right)$ and $X_{2}=R\left(A^{r}\right)$.

2. Operations on sets of operators. Let $X$ be a vector space and $L(X)$ the set of operators on $X$. In the following proposition $\operatorname{comm}(A)$ denotes the commutant of a set $A$ in $L(X)$. 
Proposition 2.1. Let $A \subseteq L(X)$ and $B \subseteq \operatorname{comm}(A)$. If $A$ and $B$ both have finite ascent and finite descent then $A \cup B$ has finite ascent and finite descent. Moreover, if $r=\alpha(A)=\delta(A), s=\alpha(B)=\delta(B)$ and $k=\alpha(A \cup B)=\delta(A \cup B)$ then $k \leq r+s$.

Proof. By Theorem 1.12, $X=X_{1} \oplus X_{2}$ where $X_{1}=N\left(A^{r}\right), X_{2}=R\left(A^{r}\right)$ and $r=\alpha(A)=\delta(A)$. Similarly, $X=Y_{1} \oplus Y_{2}$ where $Y_{1}=N\left(B^{s}\right), Y_{2}=$ $R\left(B^{s}\right)$ and $s=\alpha(B)=\delta(B)$. Let $Z_{1}=X_{1} \cap Y_{1}$ and $Z_{2}=\left(X_{1} \cap Y_{2}\right) \oplus X_{2}$. Since $B \subseteq \operatorname{comm}(A)$, both $Z_{1}$ and $Z_{2}$ are $A \cup B$-invariant. Now, $X=Z_{1} \oplus Z_{2}$ where $\left.A \cup B\right|_{Z_{1}}$ is nilpotent and $\left.A \cup B\right|_{Z_{2}}$ is bijective. Hence by Theorem 1.12, $A \cup B$ has finite ascent and finite descent. Also, by Theorem 1.12, $Z_{1}=$ $N\left((A \cup B)^{k}\right)$ and $Z_{2}=R\left((A \cup B)^{k}\right)$ where $k=\alpha(A \cup B)=\delta(A \cup B)$. Thus $N\left((A \cup B)^{k}\right)=N\left(A^{r}\right) \cap N\left(B^{s}\right) \subseteq N\left((A \cup B)^{r+s}\right)$. By Proposition 1.9, the ascending chain length of $A \cup B$ is $k$ and so $k \leq r+s$.

Note that if we remove the condition $B \subseteq \operatorname{comm}(A)$ then the conclusion to Proposition 2.1 will not hold in general. Also, the converse to Proposition 2.1 does not hold.

EXAMPLE 2.2 .

(i) Consider the $2 \times 2$ matrices $a=\left(\begin{array}{ll}0 & 1 \\ 0 & 0\end{array}\right)$ and $b=\left(\begin{array}{ll}0 & 0 \\ 0 & 1\end{array}\right)$. We have $\alpha(a)=\delta(a)=2$ and $\alpha(b)=\delta(b)=1$. However, $\operatorname{acl}(\{a, b\})=1$, $\operatorname{dcl}(\{a, b\})=\delta(\{a, b\})=0$ and $\alpha(\{a, b\})=\infty$.

(ii) Let $X=\operatorname{span}\left\{e_{i, j}: i, j \in \mathbb{N}_{0}\right\}$ and $A=\left\{a_{1}, a_{2}\right\}$ where

$$
a_{1}\left(e_{i, j}\right)=\left\{\begin{array}{ll}
e_{i-1, j} & \text { if } i>0, \\
0 & \text { if } i=0,
\end{array} \quad \text { and } \quad a_{2}\left(e_{i, j}\right)=e_{i, j+1} .\right.
$$

Since $a_{1}$ is surjective and $a_{2}$ is injective we have $\alpha(A)=\delta(A)=0$. However, the ascent of $a_{1}$ is infinite and the descent of $a_{2}$ is infinite.

Denote by $\langle A\rangle$ the algebra generated by a subset $A$ in $L(X)$.

Proposition 2.3. Let $A \subseteq L(X)$ be a non-empty set. Then

(i) $\operatorname{acl}(A)=\operatorname{acl}(\langle A\rangle)$ and $\operatorname{dcl}(A)=\operatorname{dcl}(\langle A\rangle)$,

(ii) $\alpha(A)=\alpha(\langle A\rangle)$ and $\delta(A)=\delta(\langle A\rangle)$.

Proof. Note that $N\left(A^{k}\right)=N\left(\langle A\rangle^{k}\right)$ and $R\left(A^{k}\right)=R\left(\langle A\rangle^{k}\right)$ for each $k$.

Let $X$ be a Banach space (real or complex). If $A \subseteq B(X)$ then $\bar{A}$ denotes the closure of $A$ in the norm topology of $B(X)$.

Proposition 2.4. Let $A$ be a non-empty subset of $B(X)$. If $R\left(A^{k}\right)$ is closed for all $k \in \mathbb{N}$ then

(i) $\operatorname{acl}(A)=\operatorname{acl}(\bar{A})$ and $\operatorname{dcl}(A)=\operatorname{dcl}(\bar{A})$,

(ii) $\alpha(A)=\alpha(\bar{A})$ and $\delta(A)=\delta(\bar{A})$. 
Proof. Note that $N\left(A^{k}\right)=N\left((\bar{A})^{k}\right)$ and $\overline{R\left(A^{k}\right)}=\overline{R\left((\bar{A})^{k}\right)}$ for all $k \in \mathbb{N}$. If $R\left(A^{k}\right)$ is closed then $R\left(A^{k}\right)=R\left((\bar{A})^{k}\right)$.

3. Tuples of operators. Let $\mathbf{a}=\left(a_{1}, \ldots, a_{n}\right)$ be an $n$-tuple of operators on a vector space $X$. For our purposes the ordering on $\mathbf{a}=\left(a_{1}, \ldots, a_{n}\right)$ is not important but for clarity we adopt the following convention: for each tuple $\mathbf{a}=\left(a_{1}, \ldots, a_{n}\right)$ and each $k \in \mathbb{N}$, $\mathbf{a}^{k}$ denotes the lexicographically ordered tuple $\left(a_{i_{1}} \ldots a_{i_{k}}\right)_{i_{1}, \ldots, i_{k}=1}^{n}$ consisting of $n^{k}$ operators and $\mathbf{a}^{0}$ denotes the identity operator on $X$. Our main interest is in the kernel and range spaces of the following row and column operators:

$$
\begin{aligned}
\operatorname{row}(\mathbf{a}): X^{n} \rightarrow X, & \left(x_{1}, \ldots, x_{n}\right) \mapsto a_{1} x_{1}+\cdots+a_{n} x_{n}, \\
\operatorname{col}(\mathbf{a}): X \rightarrow X^{n}, & x \mapsto\left(a_{1} x, \ldots, a_{n} x\right) .
\end{aligned}
$$

The terminology and notation for sets of operators introduced thus far will also be used for tuples $\mathbf{a}=\left(a_{1}, \ldots, a_{n}\right)$. In this case $\mathbf{a}=\left(a_{1}, \ldots, a_{n}\right)$ may be substituted by the underlying subset $A=\bigcup_{j=1}^{n}\left\{a_{j}\right\}$ in $L(X)$. For example, $\alpha(\mathbf{a})=\alpha(A)$ and $\delta(\mathbf{a})=\delta(A)$. For each $k \in \mathbb{N}$ we write $N\left(\mathbf{a}^{k}\right):=\operatorname{Ker} \operatorname{col}\left(\mathbf{a}^{k}\right)=N\left(A^{k}\right)$ and $R\left(\mathbf{a}^{k}\right):=\operatorname{Ran} \operatorname{row}\left(\mathbf{a}^{k}\right)=R\left(A^{k}\right)$.

Proposition 3.1. Let $\mathbf{a}=\left(a_{1}, \ldots, a_{n}\right)$ be an $n$-tuple of operators on $X$. Then

(i) $\alpha(\mathbf{a})=\min \left(\left\{r \in \mathbb{N}_{0}: \operatorname{Ker} \operatorname{row}\left(\mathbf{a}^{r}\right)=\operatorname{Ker} \operatorname{col}(\mathbf{a}) \circ \operatorname{row}\left(\mathbf{a}^{r}\right)\right\} \cup\{\infty\}\right)$,

(ii) $\delta(\mathbf{a})=\min \left(\left\{r \in \mathbb{N}_{0}: \operatorname{Ran} \operatorname{col}\left(\mathbf{a}^{r}\right)=\operatorname{Ran} \operatorname{col}\left(\mathbf{a}^{r}\right) \circ \operatorname{row}(\mathbf{a})\right\} \cup\{\infty\}\right)$.

Proof. For any composition $W \stackrel{s}{\rightarrow} X \stackrel{t}{\rightarrow} Y$ of operators $s, t$ between vector spaces $W, X$ and $Y$, we have isomorphisms $\operatorname{Ker}(t \circ s) / \operatorname{Ker} s \cong$ $\operatorname{Ker} t \cap \operatorname{Ran} s$ and $\operatorname{Ran} t / \operatorname{Ran}(t \circ s) \cong X /(\operatorname{Ker} t+\operatorname{Ran} s)$. The result follows on applying this fact to the appropriate row and column operators.

Remark 3.2. The formulae in Proposition 3.1 can be seen to coincide with the definitions of ascent and descent introduced in [2] for tuples of commuting module endomorphisms.

Proposition 3.3. Let $\mathbf{a}=\left(a_{1}, \ldots, a_{n}\right)$ be a commuting $n$-tuple of operators on a finite-dimensional vector space $X$. Then $\mathbf{a}$ has finite ascent and finite descent.

Proof. Since $X$ is finite-dimensional, each operator $a_{j}$ has finite ascent and descent. The result follows by repeated application of Proposition 2.1.

Given an $n$-tuple $\mathbf{a}=\left(a_{1}, \ldots, a_{n}\right)$ of operators on $X$ we denote by $\mathbf{a}^{\prime}=$ $\left(a_{1}^{\prime}, \ldots, a_{n}^{\prime}\right)$ the tuple of transpose operators on the algebraic conjugate $X^{\prime}$. 
Proposition 3.4. Let $\mathbf{a}=\left(a_{1}, \ldots, a_{n}\right)$ be an $n$-tuple of operators on $X$. Then

(i) $\operatorname{acl}(\mathbf{a})=\operatorname{dcl}\left(\mathbf{a}^{\prime}\right)$ and $\operatorname{dcl}(\mathbf{a})=\operatorname{acl}\left(\mathbf{a}^{\prime}\right)$,

(ii) $\alpha(\mathbf{a})=\delta\left(\mathbf{a}^{\prime}\right)$ and $\delta(\mathbf{a})=\alpha\left(\mathbf{a}^{\prime}\right)$.

Proof. Note that $\operatorname{col}\left(\mathbf{a}^{k}\right)^{\prime}=\operatorname{row}\left(\left(\mathbf{a}^{\prime}\right)^{k}\right)$ and $\operatorname{row}\left(\mathbf{a}^{k}\right)^{\prime}=\operatorname{col}\left(\left(\mathbf{a}^{\prime}\right)^{k}\right)$ for each $k \in \mathbb{N}$.

Let $X$ be a real or complex Banach space. An $n$-tuple $\mathbf{a}=\left(a_{1}, \ldots, a_{n}\right)$ of bounded operators on $X$ is called upper Fredholm if $\operatorname{col}(\mathbf{a})$ is upper Fredholm, lower Fredholm if row (a) is lower Fredholm, and Fredholm if a is both upper and lower Fredholm. (See $[3,6,9]$.) We denote by $\mathbf{a}^{*}=\left(a_{1}^{*}, \ldots, a_{n}^{*}\right)$ the $n$-tuple of adjoint operators on the dual space $X^{*}$.

Proposition 3.5. Let $\mathbf{a}=\left(a_{1}, \ldots, a_{n}\right)$ be an $n$-tuple of bounded operators on $X$. If $\mathbf{a}=\left(a_{1}, \ldots, a_{n}\right)$ is Fredholm then

(i) $\operatorname{acl}(\mathbf{a})=\operatorname{dcl}\left(\mathbf{a}^{*}\right)$ and $\operatorname{dcl}(\mathbf{a})=\operatorname{acl}\left(\mathbf{a}^{*}\right)$,

(ii) $\alpha(\mathbf{a})=\delta\left(\mathbf{a}^{*}\right)$ and $\delta(\mathbf{a})=\alpha\left(\mathbf{a}^{*}\right)$.

Proof. Note that $\operatorname{col}\left(\mathbf{a}^{k}\right)^{*}=\operatorname{row}\left(\left(\mathbf{a}^{*}\right)^{k}\right)$ and $\operatorname{row}\left(\mathbf{a}^{k}\right)^{*}=\operatorname{col}\left(\left(\mathbf{a}^{*}\right)^{k}\right)$ for each $k$. Since a is Fredholm, $R\left(\left(\mathbf{a}^{*}\right)^{k}\right)$ is weak* closed for each $k \in \mathbb{N}$. Also, $R\left(\mathbf{a}^{k}\right)$ is closed and $N(\mathbf{a})+R\left(\mathbf{a}^{k}\right)$ is closed for all $k \in \mathbb{N}$. The results now follow by elementary arguments.

An $n$-tuple $\mathbf{a}=\left(a_{1}, \ldots, a_{n}\right)$ is called bounded below if $\operatorname{col}(\mathbf{a})$ is bounded below, and surjective if $\operatorname{row}(\mathbf{a})$ is surjective.

Proposition 3.6. Let $\mathbf{a}=\left(a_{1}, \ldots, a_{n}\right)$ be an $n$-tuple of bounded operators on $X$. Then $\mathbf{a}=\left(a_{1}, \ldots, a_{n}\right)$ is Fredholm with finite ascent and finite descent if and only if there exist closed a-invariant subspaces $X_{1}, X_{2}$ of $X$ such that $X=X_{1} \oplus X_{2}, X_{1}$ is finite-dimensional, $\left.\mathbf{a}\right|_{X_{1}}$ is nilpotent and $\left.\mathbf{a}\right|_{X_{2}}$ is both bounded below and surjective.

Proof. Suppose a is Fredholm with finite ascent and descent. Then there exist subspaces $X_{1}, X_{2}$ of $X$ as in Theorem 1.12 with $X_{1}=N\left(\mathbf{a}^{r}\right), X_{2}=R\left(\mathbf{a}^{r}\right)$ and $r=\alpha(\mathbf{a})=\delta(\mathbf{a})$. Since $\mathbf{a}$ is Fredholm, $X_{1}$ is finite-dimensional and $X_{2}$ is closed. Also, $\operatorname{col}\left(\left.\mathbf{a}\right|_{X_{2}}\right)$ has closed range. Since $\left.\mathbf{a}\right|_{X_{2}}$ is injective it follows that $\left.\mathbf{a}\right|_{X_{2}}$ is bounded below.

Suppose, conversely, that there exist subspaces $X_{1}, X_{2}$ of $X$ as in the statement of the proposition. By Theorem 1.12, a has finite ascent and finite descent. That $\mathbf{a}$ is upper Fredholm follows since $X_{1}$ is finite-dimensional and $\left.\mathbf{a}\right|_{X_{2}}$ is bounded below. That $\mathbf{a}$ is lower Fredholm follows since $X_{1}$ is finite-dimensional and $\left.\mathbf{a}\right|_{X_{2}}$ is surjective. 
4. A Browder joint spectrum. Throughout this section we consider commuting tuples $\mathbf{a}=\left(a_{1}, \ldots, a_{n}\right)$ of bounded operators on an infinitedimensional complex Banach space $X$. (Note that commutativity was generally not required in the previous sections.) If $\lambda=\left(\lambda_{1}, \ldots, \lambda_{n}\right) \in \mathbb{C}^{n}$ then $\mathbf{a}-\lambda$ will denote the $n$-tuple $\left(a_{1}-\lambda_{1}, \ldots, a_{n}-\lambda_{n}\right)$. The joint approximate point spectrum is denoted by $\sigma_{\pi}$ and the joint defect spectrum is denoted by $\sigma_{\delta}$. We refer the reader to $[3,6]$ for a full treatment of joint spectra.

Definition 4.1. A commuting $n$-tuple $\mathbf{a}=\left(a_{1}, \ldots, a_{n}\right)$ of bounded operators on a complex Banach space $X$ is called a Browder tuple on $X$ if $\mathbf{a}$ is Fredholm with finite ascent and finite descent. The Browder spectrum of a commuting tuple $\mathbf{a}=\left(a_{1}, \ldots, a_{n}\right)$ on $X$ is given by

$$
\sigma_{\mathrm{b}}(\mathbf{a})=\left\{\lambda \in \mathbb{C}^{n}: \mathbf{a}-\lambda \text { not Browder }\right\} .
$$

REMARK 4.2. In the case of a single operator the above definitions coincide with that of a Browder (or Riesz-Schauder) operator and the Browder essential spectrum. (See for example $[3,5,6]$.)

Proposition 4.3. Let $\mathbf{a}=\left(a_{1}, \ldots, a_{n}\right)$ be a commuting $n$-tuple of bounded operators on $X$. Then $\mathbf{a}=\left(a_{1}, \ldots, a_{n}\right)$ is a Browder tuple on $X$ if and only if $\mathbf{a}^{*}=\left(a_{1}^{*}, \ldots, a_{n}^{*}\right)$ is a Browder tuple on $X^{*}$.

Proof. A tuple $\mathbf{a}=\left(a_{1}, \ldots, a_{n}\right)$ is Fredholm if and only if the adjoint tuple $\mathbf{a}^{*}=\left(a_{1}^{*}, \ldots, a_{n}^{*}\right)$ is Fredholm $([6$, Corollary III.19.12] $)$. Thus the result follows on applying Proposition 3.5.

Proposition 4.4. $\sigma_{\mathrm{b}}$ is compact-valued.

Proof. Let $\mathbf{a}=\left(a_{1}, \ldots, a_{n}\right)$ be a commuting tuple of bounded operators on $X$. Then $\sigma_{\mathrm{b}}(\mathbf{a}) \subseteq \sigma_{\pi}(\mathbf{a}) \cup \sigma_{\delta}(\mathbf{a})$ and so $\sigma_{\mathrm{b}}(\mathbf{a})$ is bounded. To show that $\sigma_{\mathrm{b}}(\mathbf{a})$ is closed, suppose $\lambda \notin \sigma_{\mathrm{b}}(\mathbf{a})$. Then $\mathbf{a}-\lambda$ is Fredholm with finite ascent and descent and so there exist subspaces $X_{1}, X_{2}$ as in Proposition 3.6. Since $\mathbf{a}-\left.\lambda\right|_{X_{2}}$ is bounded below and surjective, there exists $\varepsilon>0$ such that $\mathbf{a}-\left.\mu\right|_{X_{2}}$ is bounded below and surjective for all $|\lambda-\mu|<\varepsilon$. Since $X_{1}$ is finite-dimensional and $\mathbf{a}-\left.\lambda\right|_{X_{1}}$ is nilpotent, it follows that $\mathbf{a}-\mu$ is bounded below and surjective for all $0<|\lambda-\mu|<\varepsilon$. Thus $\mathbf{a}-\mu$ is a Browder tuple for all $|\lambda-\mu|<\varepsilon$ and so $\sigma_{\mathrm{b}}(\mathbf{a})$ is closed.

Proposition 4.5. $\sigma_{\mathrm{b}}$ has the projection property.

Proof. Let $\mathbf{a}=\left(a_{1}, \ldots, a_{n}\right)$ be a commuting tuple of bounded operators on $X$. We need to show that $p\left(\sigma_{\mathrm{b}}(\mathbf{a})\right)=\sigma_{\mathrm{b}}(p(\mathbf{a}))$ for all projections $p: \mathbb{C}^{n} \rightarrow$ $\mathbb{C}^{k}$ given by $p\left(z_{1}, \ldots, z_{n}\right)=\left(z_{i_{1}}, \ldots, z_{i_{k}}\right), 1 \leq i_{1}<\cdots<i_{k} \leq n$. Firstly we show the inclusion $p\left(\sigma_{\mathrm{b}}(\mathbf{a})\right) \subseteq \sigma_{\mathrm{b}}(p(\mathbf{a}))$.

Let $\lambda \in \mathbb{C}^{n}$ and suppose $p(\lambda) \notin \sigma_{\mathrm{b}}(p(\mathbf{a}))$. Then $p(\mathbf{a})-p(\lambda)=\left(a_{i_{1}}-\lambda_{i_{1}}\right.$, $\left.\ldots, a_{i_{k}}-\lambda_{i_{k}}\right)$ is Fredholm with finite ascent and finite descent. Thus there exist subspaces $X_{1}, X_{2}$ as in Proposition 3.6. Moreover, we may take $X_{1}=$ 
$N\left(B^{r}\right)$ and $X_{2}=R\left(B^{r}\right)$ where $B=\bigcup_{j=1}^{k}\left\{a_{i_{j}}-\lambda_{i_{j}}\right\}$ and $r=\alpha(B)=\delta(B)$. Since $\mathbf{a}$ is a commuting tuple, $N\left(B^{r}\right)$ and $R\left(B^{r}\right)$ are a-invariant. Now, $X_{1}$ is finite-dimensional and $\mathbf{a}-\left.\lambda\right|_{X_{2}}$ is bijective. It follows that $\mathbf{a}-\lambda$ has finite ascent and finite descent. That $\mathbf{a}-\lambda$ is Fredholm follows since $\mathbf{a}-\left.\lambda\right|_{X_{2}}$ is bounded below and surjective and $X_{1}$ is finite-dimensional. Hence $\mathbf{a}-\lambda$ is a Browder tuple and so $\lambda \notin \sigma_{\mathrm{b}}(\mathbf{a})$. We conclude that $p\left(\sigma_{\mathrm{b}}(\mathbf{a})\right) \subseteq \sigma_{\mathrm{b}}(p(\mathbf{a}))$.

For the reverse inclusion, suppose $\mu=\left(\mu_{i_{1}}, \ldots, \mu_{i_{k}}\right) \in \sigma_{\mathrm{b}}(p(\mathbf{a}))$. If $p(\mathbf{a})-\mu=\left(a_{i_{1}}-\mu_{i_{1}}, \ldots, a_{i_{k}}-\mu_{i_{k}}\right)$ is not Fredholm then by the projection property for the Fredholm spectrum (see [6, Theorem III.19.15]) there exists $\lambda \in \mathbb{C}^{n}$ with $p(\lambda)=\mu$ such that $\mathbf{a}-\lambda$ is not Fredholm. Thus $\mu \in p\left(\sigma_{\mathrm{b}}(\mathbf{a})\right)$.

Suppose that $p(\mathbf{a})-\mu$ is Fredholm with infinite ascent. Let $B=$ $\bigcup_{j=1}^{k}\left\{a_{i_{j}}-\mu_{i_{j}}\right\}$. Then the spaces $N(B) \cap R\left(B^{j}\right)$ are non-zero and form a decreasing chain. Since $p(\mathbf{a})-\mu$ is upper Fredholm, $N(B)$ is finite-dimensional. Hence there exists $r$ such that $N(B) \cap R\left(B^{r}\right)=N(B) \cap R\left(B^{s}\right)$ for all $s \geq r$. Thus $Z=N(B) \cap R\left(B^{r}\right)$ is a non-zero finite-dimensional space. Let $q$ : $\mathbb{C}^{n} \rightarrow \mathbb{C}^{n-k}$ be the complementary projection to $p$. That is, $q\left(z_{1}, \ldots, z_{n}\right)=$ $\left(z_{i_{k+1}}, \ldots, z_{i_{n}}\right)$ where $1 \leq i_{k+1}<\ldots<i_{n} \leq n$ and $i_{k+1}, \ldots, i_{n} \notin\left\{i_{1}, \ldots, i_{k}\right\}$. Since $\mathbf{a}=\left(a_{1}, \ldots, a_{n}\right)$ is a commuting tuple, $Z$ is invariant for $q(\mathbf{a})=$ $\left(a_{i_{k+1}}, \ldots, a_{i_{n}}\right)$. Thus there exists a joint eigenvalue $\omega \in \mathbb{C}^{n-k}$ for $\left.q(\mathbf{a})\right|_{Z}$ with a corresponding eigenvector $x \in Z$. Let $\lambda=\left(\lambda_{1}, \ldots, \lambda_{n}\right)$ where $p(\lambda)=\mu$ and $q(\lambda)=\omega$ and let $A=\bigcup_{j=1}^{n}\left\{a_{j}-\lambda_{j}\right\}$. Then $x$ is a non-zero element in $N(A) \cap R\left(A^{j}\right)$ for all $j \geq 0$ and so the ascent of $\mathbf{a}-\lambda$ is infinite. Thus $\lambda \in \sigma_{\mathrm{b}}(\mathbf{a})$ and so $\mu \in p\left(\sigma_{\mathrm{b}}(\mathbf{a})\right)$.

If $p(\mathbf{a})-\mu$ is Fredholm with infinite descent then by Proposition 3.5, the adjoint tuple $(p(\mathbf{a})-\mu)^{*}=\left(a_{i_{1}}^{*}-\mu_{i_{1}}, \ldots, a_{i_{k}}^{*}-\mu_{i_{k}}\right)$ is Fredholm with infinite ascent. Thus by the above argument there exists $\lambda \in \mathbb{C}^{n}$ such that $p(\lambda)=\mu$ and $(\mathbf{a}-\lambda)^{*}$ is not a Browder tuple. By Proposition 4.3, $\mathbf{a}-\lambda$ is not a Browder tuple and so $\mu \in p\left(\sigma_{\mathrm{b}}(\mathbf{a})\right)$. Thus we have shown that $p\left(\sigma_{\mathrm{b}}(\mathbf{a})\right) \supseteq \sigma_{\mathrm{b}}(p(\mathbf{a}))$.

REMARK 4.6. As a consequence of Propositions 4.4 and $4.5, \sigma_{\mathrm{b}}$ is a subspectrum in the sense of W. Żelazko ([10]) and a compact-valued spectral system in the sense of V. Müller ([6]). By a result of M. Putinar ([7]), $\sigma_{\mathrm{b}}$ satisfies a spectral mapping theorem for functions analytic on a neighbourhood of the Taylor spectrum (see also [6, Corollary IV.30.11]).

The following proposition extends a characterisation of D. C. Lay ([5]).

Proposition 4.7. Let $\mathbf{a}=\left(a_{1}, \ldots, a_{n}\right)$ be a commuting $n$-tuple of bounded operators on a complex Banach space $X$ and denote by $\mathcal{R}$ the set of commuting $n$-tuples $\mathbf{r}=\left(r_{1}, \ldots, r_{n}\right)$ of Riesz (or compact, or finite rank) 
operators on $X$ such that $a_{i} r_{j}=r_{j} a_{i}$ for all $i, j=1, \ldots, n$. Then

$$
\sigma_{\mathrm{b}}(\mathbf{a})=\bigcap_{\mathbf{r} \in \mathcal{R}} \sigma_{\pi}(\mathbf{a}+\mathbf{r}) \cup \sigma_{\delta}(\mathbf{a}+\mathbf{r}) .
$$

Proof. If $\lambda \notin \sigma_{\mathrm{b}}(\mathbf{a})$ then $\mathbf{a}-\lambda$ is Fredholm with finite ascent and descent. Thus there exist subspaces $X_{1}, X_{2}$ as in Proposition 3.6. Let $P$ be the finite rank projection onto $X_{1}$ along $X_{2}$ and let $\mathbf{r}=(P, \ldots, P)$. Then $\mathbf{r} \in \mathcal{R}$ and $\mathbf{a}+\mathbf{r}-\lambda$ is both bounded below and surjective. Hence $\lambda \notin \sigma_{\pi}(\mathbf{a}+\mathbf{r}) \cup \sigma_{\delta}(\mathbf{a}+\mathbf{r})$.

For the reverse inclusion, suppose $\lambda \notin \sigma_{\pi}(\mathbf{a}+\mathbf{r}) \cup \sigma_{\delta}(\mathbf{a}+\mathbf{r})$ for some $\mathbf{r} \in \mathcal{R}$. Then $\lambda \notin \sigma_{\mathrm{b}}(\mathbf{a}+\mathbf{r})$. Define the polynomial mapping $p: \mathbb{C}^{2 n} \rightarrow \mathbb{C}^{n}$ by $p\left(z_{1}, \ldots, z_{n}, w_{1}, \ldots, w_{n}\right)=\left(z_{1}+w_{1}, \ldots, z_{n}+w_{n}\right)$. Applying the spectral mapping theorem, we have

$$
\sigma_{\mathrm{b}}(\mathbf{a}+\mathbf{r})=p\left(\sigma_{\mathrm{b}}(\mathbf{a}, \mathbf{r})\right) \subseteq p\left(\sigma_{\mathrm{b}}(\mathbf{a}) \times \sigma_{\mathrm{b}}(\mathbf{r})\right)=p\left(\sigma_{\mathrm{b}}(\mathbf{a}) \times\{0\}\right)=\sigma_{\mathrm{b}}(\mathbf{a}) .
$$

Thus $\lambda \notin \sigma_{\mathrm{b}}(\mathbf{a})$.

Remark 4.8. Note that for the Taylor-Browder spectrum $\sigma_{\mathrm{Tb}}=\sigma_{\mathrm{Te}} \cup$ $\sigma_{\mathrm{T}}^{\prime}$ of R. E. Curto and A. T. Dash ([1]) and the upper and lower semiBrowder spectra $\sigma_{\mathrm{B}+}, \sigma_{\mathrm{B}-}$ of Kordula, Müller and Rakočević ([4]) there are inclusions, $\sigma_{\mathrm{B}+} \cup \sigma_{\mathrm{B}-} \subseteq \sigma_{\mathrm{b}} \subseteq \sigma_{\mathrm{Tb}}$. From Example 1.5 the first inclusion may be strict. The following example shows the second inclusion may also be strict:

EXAMPLE 4.9. Let $H$ be the complex Hilbert space with orthonormal basis $\left\{e_{i, j}: i, j \in \mathbb{N}_{0}\right\}$. Let $\mathbf{a}=\left(a_{1}, a_{2}\right)$ be the pair of commuting bounded operators on $H$ given by

$$
a_{1}\left(e_{i, j}\right)=\left\{\begin{array}{ll}
e_{i-1, j} & \text { if } i>0, \\
0 & \text { if } i=0,
\end{array} \quad a_{2}\left(e_{i, j}\right)=\frac{1}{2} e_{i, j+1} .\right.
$$

Then $\mathbf{a}=\left(a_{1}, a_{2}\right)$ is bounded below and surjective. Hence $(0,0) \notin \sigma_{\mathrm{b}}(\mathbf{a})$. Let $x=\sum_{i=0}^{\infty} 2^{-i} e_{i, i} \in H$. Then $a_{1} x=a_{2} x$ and $(x, x) \notin \operatorname{Ran} \operatorname{col}(\mathbf{a})$. Thus $(x, x) \in H \oplus H$ represents a non-zero element in the cohomology of the Koszul complex for a. Hence $(0,0) \in \sigma_{\mathrm{T}}(\mathbf{a})$. If $(0,0) \notin \sigma_{\mathrm{Tb}}(\mathbf{a})$ then $(0,0)$ is an isolated point in $\sigma_{\mathrm{T}}(\mathbf{a})$. Constructing the spectral projection $E$ associated to $(0,0)$ via the Taylor functional calculus we obtain a decomposition of the form in Theorem 1.12. Since a has ascent and descent 0 , it follows that $E$ must be the zero operator. But this contradicts the fact that $(0,0) \in \sigma_{\mathrm{T}}(\mathbf{a})$. Hence $(0,0) \in \sigma_{\mathrm{Tb}}(\mathbf{a})$.

Acknowledgements. This work forms part of the authors PhD Thesis carried out at Trinity College Dublin under the guidance of Richard Timoney to whom the author wishes to express his gratitude. Further thanks are due to Robin Harte for many helpful comments.

This research is based on work supported by Science Foundation Ireland under grant 05/RFP/MAT0033. 


\section{References}

[1] R. E. Curto and A. T. Dash, Browder spectral systems, Proc. Amer. Math. Soc. 103 (1988), 407-413.

[2] L. Grunenfelder and M. Omladič, Ascent and descent for finite sequences of commuting endomorphisms, Pacific J. Math. 191 (1999), 95-121.

[3] R. E. Harte, Invertibility and Singularity for Bounded Linear Operators, Dekker, 1988.

[4] V. Kordula, V. Müller and V. Rakočević, On the semi-Browder spectrum, Studia Math. 123 (1997), 1-13.

[5] D. C. Lay, Characterizations of the essential spectrum of F. E. Browder, Bull. Amer. Math. Soc. 74 (1968), 246-248.

[6] V. Müller, Spectral Theory of Linear Operators and Spectral Systems in Banach Algebras, 2nd ed., Birkhäuser, 2007.

[7] M. Putinar, Functional calculus and the Gelfand transformation, Studia Math. 79 (1984), 83-86.

[8] A. E. Taylor, Theorems on ascent, descent, nullity and defect of linear operators, Math. Ann. 163 (1966), 18-49.

[9] A. E. Taylor and D. C. Lay, Introduction to Functional Analysis, 2nd ed., Wiley, 1980.

[10] W. Żelazko, An axiomatic approach to joint spectra I, Studia Math. 64 (1979), 249-261.

School of Mathematics

Trinity College

Dublin 2, Ireland

E-mail: dk@maths.tcd.ie

Received March 11, 2008

Revised version September 30, 2008 